\title{
O Método Do-It: Um Relato de Experiência sobre o Ensino de Mapeamento Sistemático da Literatura para Alunos de Graduação
}

\author{
Pasqueline Dantas Scaicoㅁ, Alexandre Scaico ${ }^{1}$, José Samuel Assunção Luz ${ }^{1}$, Juliana \\ Aragão $^{1}$
}

${ }^{1}$ Departamento de Ciências Exatas Universidade Federal da Paraíba- Rio Tinto, PBBrasil

\{pasqueline, alexandre, jose.samuel, julianaaraujo\} @dcx.ufpb.br

\begin{abstract}
After years teaching courses of introduction to research, we understand that many undergraduate students fail to develop skills to systematize literature reviews processes properly. This paper describes the method Do-It, designed to support how students might learn how to perform systematic mappings. Its pedagogical actions include use of practices of active learning, cooperative work and situated learning. Some anecdotal evidence that emerged from applying Do-It suggests that it might be efficient way for teaching this content. The method is presented along with the description of its first application in a course of introduction to research methods. The steps of Do-It are presented intertwined with its application in a project involving 24 students. Finally, a summary of some findings that was arisen from the systematic review and the teaching experience are also presented.
\end{abstract}

Resumo. Anos de experiência lecionando disciplinas introdutórias de pesquisa científica revelaram que muitos alunos na graduação não conseguem desenvolver competências para sistematizar adequadamente revisões da literatura. Neste artigo é descrito o método Do-It, elaborado com a finalidade de ensinar estudantes a como executar mapeamentos sistemáticos. As ações pedagógicas contemplam o uso de práticas ativas, trabalho cooperativo e aprendizagem situada. Algumas evidências anedóticas sugerem que este pode ser um modelo eficiente para que professores ensinem este conteúdo. $O$ método é apresentado a partir de um relato de experiência de sua aplicação em uma disciplina introdutória de pesquisa científica. Os passos do método são apresentados de maneira intercalada com a sua aplicação em um projeto formado por 24 estudantes. Um resumo dos achados da aplicação do método $e$ do que os estudantes alcançaram com o mapeamento também são apresentados neste artigo.

\section{Introdução}

Inúmeros jovens que chegam ao sistema superior de ensino dominam tecnologias que estão disponíveis na Internet. Contudo, parcela significativa dos estudantes não 
desenvolverá competências para sistematizar o processo de revisar $^{1}$ a literatura com qualidade científica, mesmo quando em estágios avançados da sua formação acadêmica. Este problema foi destacado por Liu e Houdek (2006), quando, há mais de uma década, já refletiam como os universitários desconheciam bases científicas de dados e não eram capazes de avaliar a confiabilidade da fonte de algumas publicações. Como consequência, ao incluir em suas leituras artigos que não haviam passado por algum processo de revisão mais rigoroso, a credibilidade do que aprendiam durante o processo de revisar a literatura poderia ficar comprometida. Em suas discussões, os autores destacavam também que a importância de desenvolver esta competência na formação de estudantes ainda na graduação era subestimada, o que impactava a experiência e desempenho de muitos que optavam por continuar a carreira acadêmica em programas de pós-graduação. Okoli $(2019$, p. 1) acrescenta que:

"Muitos pesquisadores não são bem treinados na realização de uma revisão de literatura autônoma, um documento acadêmico que resume e sintetiza na sua totalidade o conhecimento anterior de um corpo de pesquisa. [...] a revisão de literatura é provavelmente a metodologia de pesquisa mais utilizada, já que praticamente todos precisam preparar uma para quase todos os trabalhos que publicam. Assim, muitas vezes existe uma falsa sensação de preparação para a considerável tarefa em mãos até que comecem e percebam a enormidade do projeto. Por outro lado, o treinamento de estudantes de pós-graduação na escrita de revisões de literatura é bastante esparso [...]."

Ao longo dos anos, pôde-se notar a presença deste problema no curso de Licenciatura em Computação da Universidade Federal da Paraíba (UFPB). Ao observar várias turmas de uma disciplina introdutória de pesquisa científica, verificou-se que os estudantes têm dificuldade de perceber a importância de sistematizar um processo para "ler" um recorte da literatura; de aprender os processos existentes e diferenciá-los. Desenvolver esta competência demanda tempo e esforço, já que é comum a necessidade da leitura de um volume considerável de material. Isto afeta negativamente o engajamento dos estudantes que não estão acostumados ou não gostam de ler, especialmente, quando não são artigos que os interessam intrinsecamente. Ainda há um ponto importante: nem sempre a carga horária das disciplinas permite que este tópico seja ensinado da forma ideal. Muitos concluem a disciplina com uma visão teórica e pouco situada dos processos que envolvem revisar sistematicamente a literatura. Os impactos disso podem variar, mas um deles se repete com frequência quando se observa os estudantes que estão no final da formação no nosso curso: a dificuldade que alguns concluintes enfrentam para realizar seus trabalhos de conclusão de curso. Nem sempre por inabilidade técnica, mas, às vezes, porque não dominam técnicas sistematizadas para revisar a literatura na área na qual pretendem atuar, o que os fazem demorar muito mais na fase de compreender um problema de pesquisa que pode definir o escopo dos seus trabalhos. Assim, é no final do curso quando alguns compreendem a necessidade de aprender uma técnica, muitas vezes a pedido do orientador, para que possam definir adequadamente questões de pesquisa ou mesmo entender adequadamente os trabalhos que estão relacionados ao que estão propondo.

Muitos cursos superiores de Computação ofertam até a primeira metade do curso, pelo menos, um componente curricular que visa apresentar conceitos e técnicas

\footnotetext{
${ }^{1} \mathrm{O}$ termo revisar, neste artigo, foi utilizado de forma ampla, de maneira a representar qualquer método de revisão sistemática da literatura, seja revisões, mapeamentos sistemáticos ou mesmo meta-análises.
} 
de como produzir conhecimento científico. Segundo as diretrizes curriculares de cursos da área de Computação (BRASIL, 2016), o desenvolvimento do pensamento científico é um dos pilares essenciais para o avanço tecnológico. Aprender a revisar a literatura com o objetivo de identificar evidências fortes em uma determinada área é uma competência importante a ser desenvolvida neste processo.

Na disciplina Pesquisa Aplicada à Computação (PAC), que possui carga horária de 60 horas, o ensino de técnicas sistemáticas para revisar a literatura constitui cerca de $20 \%$ da disciplina. Segundo a ementa deste componente, no tocante a este tópico, espera-se que o estudante aprenda a usar bases científicas de dados, sistematizar o processo de busca de publicações e, dentre outros aspectos, compreender questões éticas, como aquelas relacionadas a plágio.

A experiência dos autores lecionando há muitos anos para turmas de PAC mostrou que a abordagem expositiva intercalada com algumas tarefas práticas era ineficaz para os estudantes aprenderem a conduzir algum processo sistemático de revisão da literatura, por mais simples que fossem. Eles concluíam a disciplina informados sobre as técnicas, mas incapazes de diferenciá-las e executá-las.

Neste trabalho apresentamos um relato de experiência que se trata da aplicação de um método de ensino chamado Do-It. O Do-It vem sendo desenhado e refinado ao longo de dois anos e objetiva ensinar iniciantes em pesquisa a aprender a executar mapeamentos sistemáticos (MS). Através dele, pretende-se não apenas atingir o propósito pedagógico de informar estudantes sobre a existência de processos sistemáticos para conduzir revisões, uma vez que é importante ter conhecimento sobre outras técnicas existentes, mas, principalmente, fazê-los aprender a conduzir mapeamentos sistemáticos, tendo clareza do objetivo deste método e suas limitações.

Este artigo está organizado da seguinte forma: a Seção 2 descreve o método DoIt. Em seguida, na Seção 3 apresenta-se um pequeno recorte do que os estudantes foram capazes de atingir na disciplina através do método. Na Seção 4, é traçada uma análise crítica sobre a experiência que foi aplicar o método pela primeira vez.

\section{O método Do-It e o relato da sua primeira aplicação}

Até chegar em uma versão estruturada do método, os autores agiram como observadores e críticos de como ensinavam este tópico na disciplina. Assim, o Do-It foi concebido de maneira que se pudesse seguir os passos previstos em um mapeamento sistemático, mas introduzindo elementos que pudessem fazer os estudantes: exercitarem o pensamento crítico; o trabalho colaborativo; o estabelecimento de oportunidades de aprendizagem que os incentivassem a se manter em contato com tarefas que provocassem atitudes autônomas, participativas e protagonistas; aspectos que são importantes, sobretudo, quando se compreende o poder da adoção de práticas oriundas de metodologias ativas (MARIN et al., 2010) (NIEMEYER e GERBER, 2015).

Desta maneira, o Do-It é um método simples, que se sustenta na adoção de incentivos didáticos; na minimização da exposição teórica de conceitos e na maximização de atividades práticas. Enfim, então, o que constitui a estrutura do método Do-It? Os passos de um mapeamento sistemático mais os elementos incentivadores que mencionaremos no relato. 
$\mathrm{Na}$ experiência que será relatada, 24 estudantes estavam matriculados na disciplina de Pesquisa Aplicada. Para executar o método foram necessários seis encontros, que totalizaram 12 horas. Entretanto, muitas tarefas ocorreram fora do horário da aula. Obviamente, o tempo que cada aluno precisou para se dedicar às tarefas extraclasse variou em função do ritmo individual de aprendizagem e no empenho do estudante para se dedicar a elas. Não foi objetivo da primeira aplicação do método fazer esta mensuração, mas este é um elemento importante que se pretende incorporar ao DoIt. Por que? Para que o estudante possa ter alguma noção sobre o tempo médio, por exemplo, com que colegas cumpriram as tarefas e para que o professor tenha um parâmetro para moderar o volume de tarefas semanais ou, mesmo, do tempo que pode ser dedicado a cada passo do mapeamento sistemático.

Seguindo como diretrizes os passos definidos por Kitchenham (2007) para a execução de um mapeamento sistemático, o esquema pedagógico do Do-it se ampara em onze ações. Algumas, como o leitor poderá observar, foram executadas iterativamente, através de ciclos em que os alunos revisitavam suas próprias decisões. Este elemento incentivador foi importante para fazê-los entender, por exemplo, a diferença entre um mapeamento e uma revisão sistemática. O primeiro, no caso, é uma técnica com um olhar mais quantitativo para os estudos, que permite identificar padrões ou tendências em uma área de pesquisa, enquanto o segundo, a revisão sistemática, mais qualitativo em essência. A seguir, os passos do Do-It são listados, ao mesmo tempo em que se descreve como ocorreu a sua aplicação. A saber:

Passo 1: Ensine porque revisões narrativas podem ser falhas, pouco confiáveis e enviesadas. Em laboratório, os alunos foram estimulados a fazer uma busca sobre um tópico de pesquisa. Depois estimulados a falar sobre como costumavam fazer pesquisas sobre assuntos que precisavam aprender em outras disciplinas. O professor, que atuava como um mediador semelhante ao de um grupo focal, lançava perguntas do tipo: como saber se o que você está encontrando, e que parece ser o mais recente, é realmente o mais importante nesta literatura? Como saber se outros autores trazem pontos divergentes? Por que os artigos que você encontrou não foram os mesmos encontrados por outros colegas? Como saber se você encontrou "tudo" o que era mais importante a ser capturado na literatura? Coletivamente, os alunos construíam argumentos e chegavam a respostas para os questionamentos postos e, mais importante: abriam mais questionamentos. Para tornar este momento mais dinâmico, foram distribuídos post-its para os estudantes deixarem suas impressões na lousa. Neste passo, o professor pode estimular o pensamento crítico dos estudantes, colaboração, curiosidade e protagonismo na construção de uma ideia ou opinião confrontando, por exemplo, respostas ou argumentos divergentes que podem surgir a partir dos alunos;

Passo 2: Explique o que significa sistematizar um processo de "ler" um recorte de literatura. $\mathrm{O}$ segundo momento é a oportunidade de introduzir a origem das técnicas de mapeamento e revisão sistemáticos; a área em que as técnicas surgiram e o porquê. Também é possível comentar os conceitos de protocolo e seus elementos básicos: janela temporal, chave e fontes de busca. Neste momento, tomando como base a discussão anterior sobre revisões narrativas, os autores encadeavam os argumentos e ideias dos estudantes para situar a importância desses elementos e como a decisão do seu conjunto pode ser 
subjetiva, de certa forma, porém, sempre norteada pelo objetivo que o pesquisador pretende alcançar;

Passo 3: Apresente uma plataforma para organizar as informações que serão geradas durante o MS porque é importante ensiná-los a organizar o volume de conteúdo que terão que lidar. A plataforma utilizada foi a Parsifal ${ }^{2}$. Os professores demonstraram algumas funcionalidades e pediram que os alunos criassem contas individuais. Que navegassem pelas telas para se familiarizar com as funcionalidades disponibilizadas no ambiente. Foram estimulados a explorar o Parsifal em duplas e em seguida listar em um documento compartilhado no Google Drive alguns campos que lhes chamaram atenção - ou por entenderem do que se tratavam ou mesmo o contrário. Os professores podem estimular a curiosidade dos estudantes usando o Parsifal para o que é um elemento importante de um MS, perguntando: o que precisa ser descrito no campo Questão de pesquisa;

Passo 4: Entregue um conjunto inicial de questões de pesquisa e dê atenção para que uma se volte para a busca de estudos que sejam revisões sistemáticas. Jovens que nunca tiveram contato com pesquisa têm dificuldade de estabelecer questões de pesquisa, especialmente porque definem questões que são inalcançáveis com uma técnica de revisão da literatura (seja revisão ou mapeamentos sistemáticos). No Do-It, sugere-se que o professor da disciplina entregue algumas Questões de Pesquisa (QP) como ponto de partida e explique que nem toda QP são adequadas para serem as de um MS. Esta é uma oportunidade de exercitar o pensamento crítico e a colaboração entre os estudantes. As discussões podem ajudar a reforçar a diferença entre as técnicas existentes. Como os estudantes da turma não tinham experiência foi possível perceber a dificuldade de eles classificarem QPs possíveis de serem respondidas à luz do método de MS. Ao final do encontro, sugere-se que apenas duas QP de partida sejam estabelecidas. Ao longo do processo de MS, é natural que outras sejam incluídas. A área utilizada para o exercitar o projeto de conduzir um MS foi o ensino de programação. Propõe-se no método Do-It que os alunos "saiam em busca" de estudos que sejam revisões sistemáticas. Esta é uma forma de reduzir o número de trabalhos retornados e mesmo de fazer com que eles possam entender mais sobre a técnica ao ler artigos que a mencionarão, imprescindivelmente. Logo, uma das questões do MS se concentrou na busca por estudos que fossem revisões sistemáticas. Na primeira aplicação do Do-It, as questões foram as seguintes: "QP1. Quantas revisões sistemáticas sobre o ensino de programação foram publicadas entre 2010 e 2020?" e "QP2. Quais QPs foram definidas pelos autores nas suas revisões sistemáticas?”.

Passo 5: Elabore com os estudantes uma chave de busca simples. Após discutir obstáculos que poderiam surgir ao elaborar uma chave de busca, coletivamente, os alunos estabeleceram a primeira chave. Buscou-se partir da mais simples possível: ("revisão sistemática" AND "ensino de programação"). Porém, os professores explicaram estratégias para elaboração e refinamento deste elemento do protocolo, tais como, uso de sinônimos, tesauros, coleta de

\footnotetext{
${ }^{2}$ www.parsif.al
} 
termos nas palavras-chave dos artigos relevantes retornados nas buscas - uma alusão à técnica bola de neve - e uso de operadores lógicos. Para manter o mínimo de subjetividade no mapeamento, já que o projeto estava sendo realizado por 24 alunos, manteve-se a simplicidade da chave, sem adição de termos sinônimos, por exemplo. Porém, não se deixou de esclarecer que a chave poderia ser melhorada e as implicações desta decisão, como é o caso da necessidade de reexecução das buscas para capturar estudos importantes;

Passo 6: Use apenas uma fonte de dados, sem deixar de explicar a importância de identificar outras que são relevantes. Os professores apresentaram cinco fontes ${ }^{3}$, explicando o que eram (se motores de busca ou não), destacando o que cada um disponibilizava, suas limitações e como os motores de busca tratavam diferentemente a sintaxe dos operadores lógicos. Também explicaram que alguns motores indexavam as mesmas informações, de maneira que poderia ser redundante optar pela combinação de alguns. Para simplificar, sugere-se que apenas uma base seja incluída no protocolo. Durante o projeto, o Google Acadêmico foi o motor escolhido. Esta decisão decorreu do fato da plataforma permitir realizar buscas avançadas, com a combinação de múltiplos operadores lógicos e do fato de ele indexar bases de conferências e periódicos relacionados à educação em Computação. Explicou-se a possibilidade de buscas manuais e da técnica bola de neve também como estratégia útil para identificar revisões sistemáticas relevantes;

Passo 7: Execute a primeira versão do protocolo. Cada estudante executou a busca considerando os elementos discutidos colaborativamente para compor o protocolo. O corpo de dados totalizou 120 publicações. Para tornar factível a execução do protocolo no tempo da disciplina, a cada aluno foram alocados cinco artigos. O Parsifal identifica duplicidade de publicações, mas por estarem usando contas diferentes usamos uma planilha compartilhada no Google Drive para que os estudantes identificassem as duplicatas manualmente (cada um buscou identificar se outro colega tinha alocado um artigo para o qual ele já estava alocado). Ao final deste passo, restaram 80 artigos, os quais foram redistribuídos entre os estudantes e reorganizados na planilha. Antes de lerem os artigos, foram instruídos sobre a necessidade de planejar dois novos elementos para o protocolo: os critérios de inclusão e exclusão;

Passo 8: Ajude os estudantes a pensar sobre a importância de definir critérios de inclusão. O Do-It recomenda que o professor questione os estudantes: Todos os resultados retornados pelos motores de busca devem ser incluídos? Por que? Esta discussão coletiva, mais uma vez, estimula a comunicação no grupo e a reflexão. Considerando as QPs do projeto que estabelecemos, percebemos que discussões produtivas foram encampadas pelos estudantes. Alguns acreditavam, por exemplo, que as publicações deveriam ser incluídas quando tratassem apenas do ensino de programação. Outros, que a inclusão deveria ser ampliada para menções à educação em Computação. Este

\footnotetext{
${ }^{3}$ Google acadêmico; Base de Periódicos da Capes; Renote, Revista Brasileira de Informática na Educação e o site do Congresso da Sociedade Brasileira de Computação, em especial o WEI (Workshop de Educação em Informática)
} 
momento foi importante para explicar como vieses podem ser incorporados no estudo, uma vez que este julgamento é subjetivo. Assim, planejar a estratégia de incluir os estudos foi um exercício de aprendizagem situada. Após serem instruídos sobre como aplicar estes critérios e decidir em conjunto que elementos dos estudos seriam considerados para formar um julgamento (se apenas considerando o título e resumo, por exemplo), os estudantes executaram o filtro e a planilha foi mais uma vez atualizada;

Passo 9: Questione os estudantes sobre o que deve ser excluído dos resultados. Partindo do subconjunto de artigos resultantes da etapa anterior, a necessidade de pensar sobre uma lista de critérios de exclusão foi estimulada. As discussões partiram de questionamentos feitos pelos professores. Por exemplo: Vocês acham que devem ser mantidos os artigos que não mencionam explicitamente o termo revisão sistemática? "Quem" deve decidir se o artigo é deste tipo? Será que seu colega pode fazer um julgamento diferente do seu e deixar na base artigos que você não concorda que deveriam ser mantidos? Como podemos deixar o processo menos subjetivo nesta etapa? Mais uma vez, o Do-It estimula que o estudante seja protagonista, seja emitindo opinião ou formulando argumentos. Muitos entenderam que deveriam ser excluídos artigos que não fizessem essa menção e a turma concordou que este era um critério de exclusão importante. Outros critérios foram sugeridos pelos professores - o limite de páginas e tipo do trabalho (se tese, dissertação, relatório técnico). Após esta etapa, 26 artigos foram mantidos no MS. Desta forma, basicamente, cada estudante ficou responsável pela extração de um artigo. O número de páginas de cada um deles foi considerado para compensar os dois estudantes que ficaram com dois trabalhos;

Passo 10: Guie o processo de extração dos dados. O trabalho de extração se concentrou na QP2. Porém, duas questões foram adicionadas durante o processo. Uma vez que se pretendia mapear que QPs os autores buscavam responder através das revisões sistemáticas, fazia sentido também capturar as respostas encontradas. Esta é a QP3. A QP4 estabeleceu: "Que temas mais emergiram nas RS?". Com esta questão buscou-se identificar, por exemplo, se os autores estavam interessados em investigar aspectos específicos do ensino de programação, tais como: o uso de tecnologias ou métodos de ensino de programação. Cada aluno criou um formulário de extração na sua conta do Parsifal, mais uma vez, com intuito de fazê-los compreender o potencial da plataforma. Lá fizeram a extração. Depois de todos finalizarem este passo, o corpo de dados foi transferido para a planilha compartilhada para que todos tivessem acesso às extrações individuais. Esta decisão preparou o corpo de dados para análise, uma vez que organizou os dados de maneira que os estudantes pudessem identificar mais facilmente a ocorrência de padrões;

Passo 11: Simplifique a análise, mas ensine uma técnica para que os estudantes identifiquem padrões nos dados. Porque o objetivo era exercitar, em algum grau, a análise dos dados extraídos, utilizou-se apenas a QP4 (“Que temas mais emergiram nas RS?") como mote para este propósito didático. De forma simplificada, a técnica de Análise Temática foi explicada para que os alunos entendessem como codificar os dados e identificar a ocorrência de padrões nos códigos (BRAUN; CLARK, 2006). Esta etapa do processo é a que 
requer níveis mais elaborados de pensamento crítico dos estudantes; capacidade de conectar ideias e resolver conflitos. É extremamente importante para que compreendam como evidências científicas podem ser geradas, as quais são importantes para assegurar a validade dos achados em um estudo secundário, como é caso de mapeamentos sistemáticos. Além disso, como mapeamentos têm natureza mais quantitativa, o foco na identificação de padrões ajudou os estudantes a entenderem o objetivo de conduzir um mapeamento sistemático.

\section{Resultados iniciais do MS conduzido pelos estudantes}

Os alunos conseguiram atingir resultados satisfatórios, considerando o tempo limitado para executar o MS e o fato de que estavam aprendendo a técnica. Devido à limitação de espaço do artigo, apenas alguns os achados iniciais do mapeamento conduzido pelos estudantes serão relatados, mas vale ressaltar que o uso de jogos para ensinar programação foi o tema que mais se destacou como objeto de estudo das revisões.

Os estudantes identificaram as seguintes tendências nos dados extraídos: Foram identificadas 24 revisões sistemáticas publicadas na última década (QP1). Os autores de revisões têm forte interesse em compreender que ferramentas são utilizadas por educadores que usam jogos como recurso para ensinar programação (QP2). Outro padrão observado é que a maioria dos estudos que aplicaram jogos como elemento de apoio utilizaram soluções desenvolvidas pelo próprio professor ou universidade no qual atuam. Poucos estudos utilizaram jogos comerciais. Dentre os jogos comerciais, os estudantes identificaram: CodeSpells ${ }^{4}$, Lightbot $^{5}$ e Minecraft ${ }^{6}(\mathrm{QP} 3)$.

Em relação às disciplinas em que os jogos são aplicados com mais frequência, elas são: Engenharia de Software, Introdução à programação e Pensamento Computacional. Mas também são citadas, mas com frequência bem menor, outras disciplinas como Estrutura de Dados e Redes de Computadores. Há uma compreensão geral que faltam evidências para indicar a eficácia dos jogos como recursos para aprendizagem de programação. Notou-se menos interesse em identificar através das revisões, quais mecânicas estão presentes em jogos que utilizados no processo de ensino de programação e que conceitos são abordados em jogos educativos. Já em relação às teorias pedagógicas utilizadas nos estudos, apenas duas revisões apresentaram essa informação. Mas elas deixam claro que a maioria dos estudos não indica se há uma teoria pedagógica baseando os estudos. Nos poucos estudos que explicitam teoria pedagógica podemos citar, em ordem de maior frequência: Teoria Pedagógica Construtivista e Taxonomia de Bloom. Outra revisão verificou que os estudos não se preocupam em adotar uma teoria de aprendizagem para fundamentar o desenvolvimento ou aplicação de jogos no processo de ensino (QP4).

\section{Considerações Finais}

Após a primeira aplicação do Do-It foi possível identificar alguns aspectos. Uma das primeiras observações é que os estudantes desenvolveram melhor a capacidade de

\footnotetext{
${ }^{4}$ https://codespells.org/

${ }^{5}$ https://lightbot.com/

${ }^{6} \mathrm{https}: / /$ www.minecraft.net/pt-br/
} 
diferenciar revisões de mapeamentos sistemáticos. Em turmas anteriores, porque a explicação recaía em expor conteúdo, a aprendizagem não se tornava situada. Conforme relatado no Passo 4 do Do-It, que consta na Seção 2, ao fazer com que os estudantes buscassem como alvo revisões sistemáticas, o professor da disciplina estabeleceu uma oportunidade de fazê-los pensar por que e no que elas diferem dos mapeamentos. À medida que leram os artigos, discussões sobre a diferença entre mapeamentos e revisões sistemáticas puderam ser constantemente estabelecidas durante as aulas. Além disso, de agregar mais informações. Por exemplo, sobre o que são estudos primários e secundários. Por causa da QP2, os alunos foram estimulados a pensar sobre as QPs contidas nas revisões sistemáticas que retornaram, com o propósito de fazê-los refletir sobre vários aspectos, dentre os quais, se estavam bem formuladas e, mesmo, se o que os autores rotulavam como revisões sistemáticas poderiam ser consideradas como tal, isto na ótica dos estudantes. É um ponto de partida para exercitar o pensamento argumentativo dos estudantes, os quais podem ser organizados em grupos para discutir questionamentos postos pelo docente.

Observou-se também que pela necessidade de colaborar dentro de uma equipe relativamente grande ( 24 pessoas), o professor pode estabelecer também um espaço para expor as muitas habilidades, inclusive soft skills, que precisam existir para que o projeto chegue a um resultado, como é o caso da resolução de conflitos criados por alguns estudantes. Outro ponto relevante emergiu com a experiência: os alunos foram estimulados a perceber como seus julgamentos subjetivos, e, certas vezes, guiados pela imaturidade técnica, levavam à introdução de vieses nas decisões que tomavam no momento de incluir ou excluir estudos. Assim, o professor pode utilizar esta oportunidade para explicar que a validade das evidências oriundas de um MS é influenciada pela presença de múltiplos revisores ao longo do processo, e de preferência, um com mais expertise na área, que podem ser organizados de maneira a resolver conflitos gerados nos julgamentos.

Em outros momentos em que a disciplina PAC foi ministrada, equipes de até três estudantes eram formadas. Cada uma recebia uma questão de pesquisa, que norteava as decisões no MS. Contudo, pela diversidade de temas, não era possível estabelecer aulas produtivas, em que as discussões pudessem resolver, por exemplo, os diferentes obstáculos que as equipes se deparavam e mesmo dar vazão para realizar questionamentos mais densos. Utilizar a mesma QP foi o fio condutor para estabelecer ritmo e sincronia nas discussões. Ao que parece, a experiência aqui relatada estabeleceu circunstâncias para que estudantes criem significado para algumas decisões que tomam. Também, para que contextualizem a informação que recebem. Esta construção pedagógica está alinhada a uma proposta situada de aprendizagem. Para Gudolle et. al (2012), "situar significa localizar os pensamentos e as ações das pessoas no tempo e no espaço". Para Lave e Wenger (1991), "o pensamento e a ação adquirem significado somente nas circunstâncias em que ocorrem”.

Ao final da disciplina os estudantes foram convidados voluntariamente a avaliar o método. Dos que responderam, todos relataram positivamente a abordagem no que tange a sua aprendizagem sobre o conteúdo, mas muitos pontuaram o desejo de ter utilizado apenas o Parsifal. Entretanto, em experiências prévias, o não uso de planilhas compartilhadas gerou alguns problemas. Primeiro, era difícil controlar quem realmente estava atualizando dados na plataforma. Depois, a forma com que o Parsifal disponibiliza os dados extraídos não facilita a análise, sobretudo para iniciantes. Os 
estudantes avaliaram de forma negativa aspectos relacionados ao trabalho em grupo, isto porque nem todos, uma minoria, vale dizer, não foi capaz de cumprir os prazos das tarefas. Assim, a visão dos dados era prejudicada, por ser a maior parte do tempo parcial, o que adiava o passo de extração. Algumas etapas seguintes do mapeamento precisaram ser adiadas. Este aspecto parece ter frustrado os estudantes que cumpriam as tarefas no prazo estipulado.

\section{Referências}

BRASIL. (2016) Resolução CNE/CES no 5. Diretrizes Curriculares Nacionais para os cursos de graduação na área da Computação. Diário Oficial [da República Federativa do Brasil], Brasília, DF, 16 de novembro.

Braun, V. and Clark, V. (2006) Using thematic analysis in psychology. Qualitative Research, v. 3, n. 2, p. 77-101.

Gudolle, L. S., Antonello, A. S. and Flach, L. (2012) Aprendizagem situada, participação e legitimidade nas práticas de trabalho. RAM, Rev. Adm. Mackenzie, São Paulo, v. 13, n. 1, p. 14-39.

Kitchenham, B. (2007). Guidelines for performing Systematic Literature Reviews in Software Engineering: Version 2.3. In: EBSE Technical Report. School of Computer Science and Mathematics, Keele University. Disponível em: < pdfs.semanticscholar.org/e62d/bbbbe70cabcde3335765009e94ed2b9883d5.pdf>. Acesso em: 12 de jan. 2021

Lave, J. and Wenger, E. (1991)_Situated learning: legitimate peripheral participation. Cambridge: Cambridge University Press.

Liu, C. and Houdek, R. (2006) Teaching Computer Science Graduate Students Scholarly Literature Review Techniques. Frontiers in Education Conference, San Diego, California.

Marin, M. J. S., Lima, E. F. G., Matsuyama, D. T., Silva, L. K. D., Gonzales, C., Deuzian, S. and Ilias, M. (2010) Aspectos das fortalezas e fragilidades no uso das Metodologias Ativas de Aprendizagem. Revista Brasileira de Educação Médica, 34 (1): p.13-20.

Niemeyer, D. J. and Gerber, H. R. (2015) Maker culture and Minecraft: Implications for the future of learning. Educational Media International, v. 52, n. 3, p. 216-226.

OKOLI, Chitu. Guia para realizar uma revisão sistemática da literatura. Tradução de David Wesley Amado Duarte; Revisão técnica e introdução de João Mattar. eaD em Foco, 2019;9 (1): e748. DOI: https://doi.org/10.18264/eadf.v9i1.748 